\section{Vulnerabilidade programática no controle da hanseníase: padrões na perspectiva de gênero no Estado da Bahia, Brasil}

\author{
Programmatic vulnerability in leprosy control: \\ gender-related patterns in Bahia State, Brazil
}

\author{
Vulnerabilidad programática en el control de la \\ lepra: padrones desde la perspectiva de género \\ en el Estado de Bahía, Brasil
}

\section{Resumo}

O objetivo deste estudo foi analisar indicadores operacionais de controle da hanseníase e sua tendência temporal, na perspectiva de gênero, no Estado da Bahia, Brasil, de 2001 a 2014. Trata-se de estudo de série temporal baseado em dados epidemiológicos da hanseníase no Sistema de Informação de Agravos de Notificação, com análise de regressão de Poisson por joinpoints e de regressão polinomial. Dos 40.054 casos novos de hanseníase analisados, 47,1\% dos contatos registrados não foram examinados, mas com tendência significativa de aumento, de forma mais acentuada entre as mulheres (variação percentual anual média - AAPC = 5, 6; IC95\%: 3,5; 7,7) em comparação aos homens $(A A P C=3,0 ;$ IC95\%: 0,5; 5, 6). A proporção de cura na coorte 2003-2014 foi de 85\%, com tendência de redução mais acentuada nos homens $(A A P C=-0,5$; IC95\%: -0,9; 0,0), comparados às mulheres (AAPC = -0,4; IC95\% -0,7;-0,1). A proporção de casos em abandono de tratamento no período foi de 5,5\%, com tendência de redução mais significativa entre mulheres ( $A A P C=-4,9$; IC95\%: - 8,7; - 1,1) do que em homens (AAPC = -2, 7; IC95\%: -4,4; - 1,0). Recidiva foi verificada em 3,8\% de todas as entradas do período; as mulheres apresentaram tendência de redução significativa (AAPC =-2,2; IC95\%: -3,3; - 1,0) $e$ os homens, de crescimento significativo (AAPC = 4,9; IC95\%: 2,9; 6,8). A análise por regressão polinomial foi consistente com a análise de joinpoints. $A$ hanseníase no Estado da Bahia apresenta indicadores operacionais com magnitude e tendência temporal significativas, em especial entre a população masculina. O desempenho insuficiente dos serviços de saúde em realizar a vigilância de contatos e a longitudinalidade do cuidado revelam diferentes dimensões de vulnerabilidade.

Hanseniase; Estudos de Séries Temporais; Gênero e Saúde
Eliana Amorim de Souza 1

Reagan Nzundu Boigny ${ }^{2}$

Anderson Fuentes Ferreira 2

Carlos Henrique Alencar 2

Maria Leide W. Oliveira 3

Alberto Novaes Ramos Jr. ${ }^{2}$

doi: 10.1590/0102-311X00196216

\section{Correspondência}

E. A. Souza

Núcleo Epidemiologia e Saúde Coletiva, Instituto

Multidisciplinar em Saúde, Universidade Federal da Bahia.

Rua Hormindo Barros 54, Quadra 17, Lote 58, Campus Anísio

Teixeira, Vitória da Conquista, BA 45029-094, Brasil.

amorim_eliana@yahoo.com.br

1 Instituto Multidisciplinar em Saúde, Universidade Federal da Bahia, Vitória da Conquista, Brasil.

2 Faculdade de Medicina, Universidade Federal do Ceará, Fortaleza, Brasil.

3 Universidade Federal do Rio de Janeiro, Rio de Janeiro, Brasil. 


\section{Introdução}

Ao longo das últimas três décadas, diferentes estratégias têm sido empreendidas para alcançar o controle da hanseníase como problema de saúde pública em escala global. O Brasil está entre os cinco países que não alcançaram a meta de controle proposta pela Organização Mundial da Saúde (OMS), persistindo com níveis de elevada endemicidade 1 . Com 28.761 notificações em 2015, o país ocupa o segundo lugar no mundo em número de casos novos, principalmente em dez clusters localizados em áreas das regiões Centro-oeste, Norte e Nordeste 2,3 .

Considerando a existência de meios diagnósticos efetivos para a hanseníase, assim como a disponibilidade e eficácia da polioquimioterapia, falhas operacionais nos diferentes pontos da rede de atenção à saúde 4,5 têm tido papel significativo para a persistência da elevada endemicidade. Como consequência, existe a presença de cenários de transmissão ativa de Mycobacterium leprae entre adolescentes e crianças (menores de 15 anos de idade), com diferencial entre gêneros, gerando potencialmente incapacidade e estigma 6,7,8. O Estado da Bahia, Região Nordeste do país, está situado dentro desse contexto epidemiológico de endemicidade 9 .

A vigilância de contatos intradomiciliares de casos de hanseníase tem sido definida como política pública estratégica desde os anos 1960, e tornou-se ao longo do tempo uma das ações prioritárias no Brasil 10. O diagnóstico precoce e o tratamento oportuno, além de minimizarem os danos causados ao indivíduo e à sua família, reduzem a dinâmica de transmissão da doença em territórios e comunidades com maior risco e vulnerabilidade.

Há um risco maior de transmissão da hanseníase no espaço domiciliar em relação à população geral, chegando a ser 14 vezes maior entre contatos intradomiciliares de casos multibacilares e aproximadamente duas vezes maior entre contatos de casos paucibacilares 11,12. É estratégico, portanto, o alcance de boa cobertura e qualidade das ações voltadas para o exame de contatos, a fim de reduzir a carga da doença 13. Uma vez diagnosticados casos da doença, os serviços de saúde deveriam empreender todos os esforços necessários para a garantia de atenção integral à saúde dentro da longitudinalidade do cuidado, com vistas ao alcance de objetivos que vão além da cura microbiológica da infecção.

Portanto, considerando-se o caráter de doença infecciosa crônica, o seguimento das pessoas acometidas pela hanseníase e suas famílias tem sido crítico, inclusive para a redução de eventuais "saídas" do sistema de notificação por abandono 14. Recentemente, o Ministério da Saúde recomendou também o reforço de busca ativa na população masculina e de idosos, visando ao diagnóstico de casos multibacilares 14 .

Outra condição necessária para atenção e vigilância da hanseníase é a qualificação da abordagem dos casos com recidiva da doença, definida como a expressão clínica da doença nos casos tratados regularmente com esquemas oficiais padronizados e corretamente indicados, que haviam recebido alta por cura, mas que apresentaram novamente sinais e sintomas clínicos de doença infecciosa ativa 15 . A recidiva remete à falha terapêutica, sendo que o Brasil foi responsável por 47,7\% (1.452) das ocorrências entre os 46 países que notificaram casos que tiveram como modo de entrada a recidiva no ano de 20152

A análise integrada de indicadores operacionais da hanseníase, incluindo análise das coortes de contatos examinados entre os registrados, de cura e de abandono, além da proporção de recidiva, permite avaliar a qualidade dos serviços de saúde no desenvolvimento de ações de controle, no sentido de gerar evidências que possibilitem a adoção de ações para superar questões operacionais do controle.

Essas questões operacionais que se expressam no Sistema Único de Saúde (SUS) podem ser a tradução de problemas relativos à universalização do acesso, integralidade da atenção, equidade, descentralização da gestão, hierarquização dos pontos de atenção, humanização e controle/participação social. A burocratização de atividades sensíveis como a abordagem de famílias em territórios representa um dos elementos críticos, por exemplo. A implantação desse sistema está, portanto, atrelada à mudança do modelo assistencial e da organização dos serviços, com base em medidas culturalmente aceitáveis dentro das reais necessidades da população.

A vulnerabilidade programática está associada a recursos sociais necessários, efetivos e democráticos, para que as pessoas de fato tenham condições de prevenir doenças ou suas complicações 16 . Remete-se também ao grau de relevância dada ao problema no âmbito da gestão, do planejamento, do monitoramento e da avaliação das ações para controle. Para tanto, as redes de atenção à saúde devem 
estar estruturadas e integradas, em perspectivas municipal e regional, com pactuação e participação social para tomada de decisões 4,17 .

Persistem lacunas quanto à possível existência de padrões diferenciais entre gênero para a avaliação de contatos intradomiciliares, alcance de alta por cura, abandono e registros de recidivas, reiterando a vulnerabilidade nessas populações. Reconhecendo que desigualdades de gênero influenciam o padrão de morbimortalidade 18, ampliando vulnerabilidades individuais, sociais e programáticas, o presente trabalho tem como objetivo analisar indicadores operacionais de controle da hanseníase e sua tendência temporal, na perspectiva de gênero, no Estado da Bahia de 2001 a 2014.

\section{Método}

\section{Local do estudo}

O Estado da Bahia é o maior da Região Nordeste e o quarto do país, com população estimada para 2015 de 15.203 .934 e densidade demográfica de 24,46 habitantes $/ \mathrm{km}^{2}$. Aproximadamente $30 \%$ da sua população vivem em zona rural de um dos seus 417 municípios (Instituto Brasileiro de Geografia e Estatística. População residente, Bahia 2014. http://tabnet.datasus.gov.br/cgi/tabcgi.exe?ibge/cnv/ popba.def, acessado em 15/Set/2016). Do ponto de vista econômico, tem o 8o Produto Interno Bruto (PIB) brasileiro, no entanto, com elevada concentração de renda revelada por sua 4a posição no país pelo índice de Gini de 0,559 19. A região nordeste do estado concentra os municípios com pelo menos três doenças negligenciadas concomitantemente, justificadas pelos mais baixos indicadores socioeconômicos 20 .

\section{Desenho de estudo}

Estudo de série temporal com base em dados secundários valendo-se de registros da notificação compulsória de casos de hanseníase no período de 2001 a 2014, de residentes no Estado da Bahia. A fonte de dados secundários teve como referência o Sistema de Informação de Agravos de Notificação - Ministério da Saúde (SINAN-MS), cuja base foi obtida junto à Diretoria de Informação em Saúde do Estado da Bahia (Secretaria de Estado da Saúde). Foram excluídos todos os casos que tiveram como critério de saída "erro diagnóstico". Por sua vez, os dados populacionais foram obtidos no Instituto Brasileiro de Geografia e Estatística (IBGE) com base nos censos populacionais do estado (2000 e 2010), e nas estimativas populacionais para os anos intercensitários (2001-2009 e 2011-2014).

\section{Análise estatística}

Inicialmente, foi verificada a frequência absoluta e relativa de casos novos de hanseníase de 2001 a 2014, considerando-se o modo de detecção: demanda espontânea, encaminhamento, exame de coletividade e exame de contato intradomiciliar.

Para compor a série história dos indicadores operacionais da hanseníase foram calculados neste estudo: (1) proporção de contatos examinados entre os contatos registrados de casos novos da coorte; (2) proporção de cura entre os casos novos da coorte; (3) proporção de casos em abandono de tratamento da coorte; e (4) proporção de casos com modo de entrada "recidiva" entre os casos notificados no ano. A análise foi estratificada segundo sexo. Utilizou-se para essa fase inicial de análise dentro do estudo o software TabWin versão 4.2 (Departamento de Informática do SUS. http://portal.saude.gov. $\mathrm{br} / \mathrm{portal} / \mathrm{se} /$ datasus/area.cfm?id_area=732).

Para a análise de tendência temporal reduzindo potenciais variabilidades da série histórica, usouse técnicas de regressão joinpoint (por pontos de inflexão) de Poisson (Joinpoint Regression Program versão 4.4.2, http://surveillance.cancer.gov/joinpoint/), e de regressão polinomial por meio do software Stata, versão 11.2 (StataCorp LP, College Station, Estados Unidos).

A regressão joinpoint proporciona o ajuste de uma série de linhas, bem como de seus pontos de inflexão em uma escala logarítmica por meio do teste de tendências anuais. Para a obtenção do ajuste baseado na melhor linha de cada segmento analisado, utilizou-se o método de permutação de Monte 
Carlo como teste de significância. A partir da definição dos seguimentos, estimou-se e testou-se a variação percentual anual (annual percentual change - APC) e a variação percentual anual média (average annual percentual change - AAPC), com seus respectivos intervalos de 95\% de confiança (IC95\%). Caso fosse verificada a ocorrência de um ponto de inflexão com sentido invertido, procedeu-se à análise em separado dos períodos do estudo.

O número de inflexões utilizadas na análise foi o resultado de modelos definidos pelo próprio programa, de forma a permitir a melhor representação da tendência, com o menor número de pontos de inflexões. O resultado possibilitou demonstrar crescimento (valores de APC positivos), redução (valores de APC negativos) ou manutenção (valor de APC igual a zero) da tendência ao longo de toda a série histórica analisada (2001-2014) 21. Considerou-se tendência estatisticamente significativa, diferente de zero, quando o valor de p era menor que 0,05.

Para a regressão polinomial, foram construídos inicialmente diagramas de dispersão entre os indicadores analisados e os anos de estudo. Seguiu-se a análise com a construção de modelos com o objetivo principal de identificar a curva que melhor se ajustava aos dados em análise, de modo a descrever a relação entre a variável dependente y (indicadores operacionais em análise) e a variável independente $\mathrm{X}$ (ano). Com vistas a prevenir a colinearidade entre os termos da equação de regressão composta, usou-se o artifício de centralizar a variável ano, transformando-a em ano calendário menos o ponto médio da série histórica em questão. As tendências foram consideradas estatisticamente significativas quando os modelos apresentaram valor de p menor que 0,05.

O estudo foi baseado em dados secundários e respeitou a Resolução no 466, de 12 de outubro de 2012 do Conselho Nacional de Saúde, e foi aprovado pelo Comitê de Ética em Pesquisa da Universidade Federal do Ceará, sob o parecer no 544.962, de 28 de fevereiro de 2014.

\section{Resultados}

No período de 14 anos do estudo, o Estado da Bahia notificou 40.054 casos novos de hanseníase, a partir dos quais estavam relacionados 104.142 mil contatos registrados. Desse total, 47,1\% (49.026) não foram examinados ao longo da coorte. A proporção de contatos examinados na coorte de 2003 a 2014 variou de 44,7\% em 2003 a 68,9\% em 2014, este último o maior percentual da série histórica (Figura 1). Verificou-se ainda tendência significativa de incremento na proporção de contatos examinados entre os registrados (AAPC = 5,3; IC95\%: 3,4; 7,2, Tabela 1), uma tendência crescente e constante $\left(y=9,9493-0,7316 x+0,0098 x^{2} ; R^{2}=0,8818 ; p<0,01\right)$, como demonstrado na Tabela 2 .

O modo de detecção de casos novos, com base em exames de contato, apresentou proporção inferior ao modo de entrada por meio de encaminhamentos e por demanda espontânea, representando 10,5\% (4.207 casos) do total. Verificou-se ainda redução do percentual de casos diagnosticados a partir da avaliação de contatos, reduzindo de 18,3\% em 2004 para 8,3\% em 2014.

A proporção de cura na coorte de 2003 a 2014 foi de $85 \%$, com a maior proporção $(87,7 \%)$ em 2009 e a menor (81\%) em 2013 (Figura 2). A tendência geral no Estado da Bahia é de redução significativa (AAPC = -0,4; IC95\%: -0,8; -0,1) (Tabela 1), com um padrão decrescente e constante $\left(y=-146431,1+5219,863 x-61,9853 x^{2}+0,2452 x^{3} ; R^{2}=0,6075 ; p=0,0483\right)$ (Tabela 2).

No total, 2.730 pessoas abandonaram o tratamento ao longo da coorte de 2003 a 2014, 5,5\% dos casos analisados, com redução dos porcentuais ao longo da série histórica, de 7,2\% em 2003 até 1,2\% em 2014 (Figura 2). Verificou-se tendência de redução a partir de 2008 (APC = -7,1; IC95\%: $-10,5 ;-3,7)$ (Tabela 1), com um padrão geral decrescente e constante (y = -303,2903+173,8827x-31,7 $\left.8731 \mathrm{x}^{2}+1,8685 \mathrm{x}^{3} ; \mathrm{R}^{2}=0,7728 ; \mathrm{p}=0,0059\right)$ (Tabela 2).

Ao longo do período de estudo foram notificados 1.537 casos como sendo recidiva da doença entre todas as modalidades de entrada do período (3,8\%), com proporção variando de 2,2\% em 2001 a $5 \%$ em 2014 (Figura 3). A tendência é de crescimento significativo ao longo do tempo, com AAPC para o estado de 4,2 (IC95\%: 1,8; 6,7) (Tabela 1) e um padrão crescente e constante (y = -15,0868+4,1240x; $\mathrm{R}^{2}=0,5896 ; \mathrm{p}=0,0013$ ) (Tabela 2).

A análise dos indicadores operacionais por sexo demonstrou que, em ambos os sexos, cresce de forma significativa a proporção de contatos examinados (Figura 1), porém de forma mais acentuada quando o caso de hanseníase registrado é uma mulher (AAPC $=5,6$; IC95\%: 3,5; 7,7) do que quando é 


\section{Figura 1}

Proporção de contatos examinados de casos novos de hanseníase nos anos da coorte, por sexo. Estado da Bahia, Nordeste do Brasil, $2003-2014$.

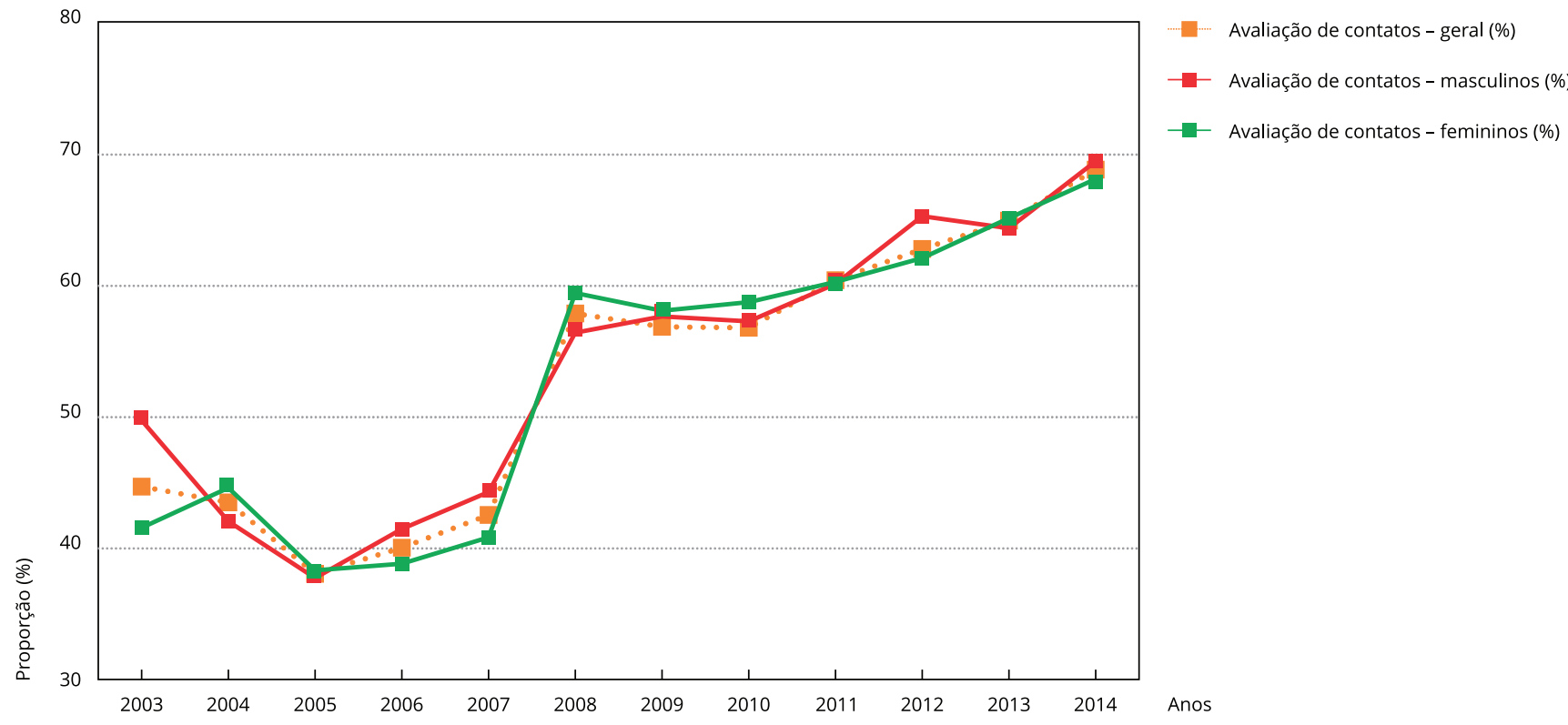

Tabela 1

Análise de tendências temporais por regressão por pontos de inflexão (joinpoints) de indicadores operacionais da hanseníase segundo sexo. Estado da Bahia, Nordeste do Brasil, 2001-2014.

\begin{tabular}{|c|c|c|c|c|c|c|}
\hline \multirow[t]{2}{*}{ Indicador } & \multirow[t]{2}{*}{ Sexo } & \multicolumn{3}{|c|}{ Tendência 1} & \multicolumn{2}{|c|}{ Período inteiro } \\
\hline & & Período & APC & IC95\% & AAPC & IC95\% \\
\hline \multirow{5}{*}{$\begin{array}{l}\text { Proporção de contatos examinados de casos novos de } \\
\text { hanseníase por sexo }\end{array}$} & M & $2003-2005$ & $-14,0$ * & $-22,6 ;-4,6$ & 3,0 * & 0,$5 ; 5,6$ \\
\hline & & $2005-2008$ & 13,9 * & 2,$6 ; 26,5$ & & \\
\hline & & $2008-2014$ & 4,1 * & 2,$3 ; 5,9$ & & \\
\hline & $\mathrm{F}$ & $2003-2014$ & 5,6 * & 3,$5 ; 7,7$ & 5,6 * & 3,$5 ; 7,7$ \\
\hline & Geral & 2003-2014 & 5.3 * & 3,$4 ; 7,2$ & $5.3 *$ & 3,$4 ; 7,2$ \\
\hline \multirow{3}{*}{$\begin{array}{l}\text { Proporção de cura entre os casos novos diagnosticados } \\
\text { nos anos das coortes }\end{array}$} & M & $2003-2014$ & $-0,5 *$ & $-0,9 ; 0,0$ & $-0,5 *$ & $-0,9 ; 0,0$ \\
\hline & $\mathrm{F}$ & $2003-2014$ & $-0,4$ * & $-0,7 ;-0,1$ & $-0,4$ * & $-0,7 ;-0,1$ \\
\hline & Geral & 2003-2014 & $-0,4^{*}$ & $-0,8 ;-0,1$ & $-0,4$ * & $-0,8 ;-0,1$ \\
\hline \multirow{5}{*}{$\begin{array}{l}\text { Proporção de abandono casos novos diagnosticados nos } \\
\text { anos das coortes }\end{array}$} & M & 2003-2014 & $-2,7^{*}$ & $-4,4 ;-1,0$ & $-2,7^{*}$ & $-4,4 ;-1,0$ \\
\hline & $\mathrm{F}$ & $2003-2008$ & 0,9 & $-6,7 ; 9,2$ & $-4,9 *$ & $-8,7 ;-1,1$ \\
\hline & & $2008-2014$ & $-9,6$ * & $-14,8 ;-4,0$ & & \\
\hline & Geral & $2003-2008$ & 0,7 & $-4,1 ; 5,7$ & $-3,6 *$ & $-6,0 ;-1,2$ \\
\hline & & $2008-2014$ & $-7,1 *$ & $-10,5 ;-3,7$ & & \\
\hline \multirow{3}{*}{$\begin{array}{l}\text { Proporção de casos com modo de entrada "recidiva" } \\
\text { entre os casos notificados no ano }\end{array}$} & M & 2001-2014 & 4,9 * & 2,$9 ; 6,8$ & 4,9 * & 2,$9 ; 6,8$ \\
\hline & $\mathrm{F}$ & $2001-2014$ & $-2,2 *$ & $-3,3 ;-1,0$ & $-2,2 *$ & $-3,3 ;-1,0$ \\
\hline & Geral & $2001-2014$ & 4,2 * & 1,$8 ; 6,7$ & 4,2 * & 1,$8 ; 6,7$ \\
\hline
\end{tabular}

AAPC: variação percentual anual média; APC: variação percentual anual; F: feminino; IC95\%: intervalo de 95\% de confiança; M: masculino.

* Significativamente diferente de $0(p<0,05)$. 


\section{Tabela 2}

Análise de tendências temporais por regressão polinomial de indicadores operacionais da hanseníase segundo sexo. Estado da Bahia, Nordeste do Brasil, 2001-2014.

\begin{tabular}{|c|c|c|c|c|c|}
\hline Indicador & Sexo & Modelo * & $\mathbf{R 2}$ & Valor de $p$ & Tendência \\
\hline $\begin{array}{l}\text { Proporção de contatos } \\
\text { examinados entre }\end{array}$ & $\mathrm{M}$ & $y=41,881+0,4758 x+0,1637 x^{2}$ & 0,8711 & 0,0006 & $\begin{array}{l}\text { Crescente e } \\
\text { constante }\end{array}$ \\
\hline \multirow[t]{2}{*}{$\begin{array}{l}\text { registrados de casos novos } \\
\text { de hanseníase }\end{array}$} & $\mathrm{F}$ & $y=19,4603-1,1128 x+0,0135 x^{2}$ & 0,8869 & 0,0001 & $\begin{array}{l}\text { Crescente e } \\
\text { constante }\end{array}$ \\
\hline & Geral & $y=9,9493-0,7316 x+0,0098 x^{2}$ & 0,8818 & 0,0001 & $\begin{array}{l}\text { Crescente e } \\
\text { constante }\end{array}$ \\
\hline Proporção de cura entre os & M & $y=-88125,9+3170,434 x-37,9872 x^{2}+0,1515 x^{3}$ & 0,6050 & 0,0495 & Decrescente \\
\hline casos novos diagnosticados & $\mathrm{F}$ & $y=-172367,5+6102,946 x-71,9900 x^{2}+0,2829 x^{3}$ & 0,5146 & 0,1066 & Decrescente \\
\hline nos anos das coortes & Geral & $y=-146431,1+5219,863 x-61,9853 x^{2}+0,2452 x^{3}$ & 0,6075 & 0,0483 & Decrescente \\
\hline $\begin{array}{l}\text { Proporção de abandono } \\
\text { entre os casos novos }\end{array}$ & M & $y=21,2611-3,4932 x$ & 0,5417 & 0,0064 & $\begin{array}{c}\text { Decrescente e } \\
\text { constante }\end{array}$ \\
\hline \multirow[t]{2}{*}{$\begin{array}{l}\text { diagnosticados nos anos } \\
\text { das coortes }\end{array}$} & $\mathrm{F}$ & $y=14,0578-2,3946 x$ & 0,6732 & 0,0011 & $\begin{array}{c}\text { Decrescente e } \\
\text { constante }\end{array}$ \\
\hline & Geral & $y=-303,2903+173,8827 x-31,78731 x^{2}+1,8685 x^{3}$ & 0,7728 & 0,0059 & $\begin{array}{c}\text { Decrescente e } \\
\text { constante }\end{array}$ \\
\hline $\begin{array}{l}\text { Proporção de casos com } \\
\text { modo de entrada "recidiva" }\end{array}$ & M & $y=-15,2996-3,5222 x$ & 0,7225 & 0,0001 & $\begin{array}{l}\text { Crescente e } \\
\text { constante }\end{array}$ \\
\hline entre os casos notificados & $\mathrm{F}$ & $y=-22,2533-15,5942 x-2,581129 x^{2}$ & 0,2736 & 0,1723 & Estável \\
\hline no ano & Geral & $y=-15,0868+4,1240 x$ & 0,5896 & 0,0013 & $\begin{array}{l}\text { Crescente e } \\
\text { constante }\end{array}$ \\
\hline
\end{tabular}

F: feminino; M: masculino; R2: coeficiente de determinação.

* Modelo: $y$ = indicador específico; $x$ = ano - ano médio do período estudado.

um homem (AAPC = 3,0; IC95\%: 0,5; 5,6), como demonstrado na Tabela 1. A Tabela 2 indica o caráter crescente e constante ao longo do tempo para ambos os sexos (homens: $y=41,881+0,4758 x+0,1637 x^{2}$; R2 0,8711; p < 0,001 - mulheres: $y=19,4603-1,1128 x+0,0135 x^{2} ; R^{2}$ 0,8869; p < 0,001). Entretanto, a análise por joinpoints revela mudança da tendência ao longo da série histórica com redução significante até 2005 e aumento a partir deste ano (Tabela 1). A redução na proporção de cura entre os casos novos de hanseníase é significativa em ambos os sexos (Figura 2), porém, com redução mais acentuada nos homens (AAPC = -0,5; IC95\%: -0,9;-0,0) do que dentre as mulheres (AAPC = -0,4; IC95\%: -0,7; -0,1) (Tabela 1). Em linhas gerais, há uma tendência decrescente ao longo da série histórica (homens: $\mathrm{y}=-88125,9+3170,434 \mathrm{x}-37,9872 \mathrm{x}^{2}+0,1515 \mathrm{x}^{3} ; \mathrm{R}^{2}=0,6050 ; \mathrm{p}=0,0495$ - mulheres: $\left.y=-172367,5+6102,946 x-71,9900 x^{2}+0,2829 x^{3} ; R^{2}=0,5146 ; p=0,1066\right)$ (Tabela 2). A tendência de queda do abandono na coorte é visualizada tanto em mulheres (AAPC = -4,9; IC95\%: $-8,7 ;-1,1$ ) quanto entre os homens (AAPC = -2,7; IC95\%: -4,4; -1,0). Em ambos os sexos a tendência de queda é verificada a partir do ano de 2008 (Tabela 1). De uma forma geral, verifica-se ao longo do período de análise uma tendência decrescente e constante (homens: $y=21,2611-3,4932 x ; R^{2}=0,5417 ; \mathrm{p}=$ 0,0064 - mulheres: $\left.y=14,0578-2,3946 x ; R^{2}=0,6732 ; p=0,0011\right)$ (Tabela 2). Para recidiva, o comportamento verificado é distinto, com as mulheres apresentando redução significativa (AAPC = -2,2; IC95\%: $-3,3 ;-1,0$ ) e os homens crescimento significativo durante toda a série histórica (AAPC = 4,9; IC95\%: 2,9; 6,8) (Tabela 1). A regressão polinomial por sua vez mostra uma tendência crescente e constante de estabilidade para o sexo masculino $\left(\mathrm{y}=-15,2996-3,5222 \mathrm{x} ; \mathrm{R}^{2}=0,7225 ; \mathrm{p}<0,001\right)$ e de estabilização para o feminino $\mathrm{y}=-22,2533-15,5942 \mathrm{x}-2,581129 \mathrm{x}^{2} ; \mathrm{R}^{2}=0,2736 ; \mathrm{p}=0,1723$ ). 


\section{Figura 2}

Proporção de cura e abandono de casos de hanseníase nos anos da coorte por sexo. Estado da Bahia, Nordeste do Brasil, $2003-2014$.

2a) Cura

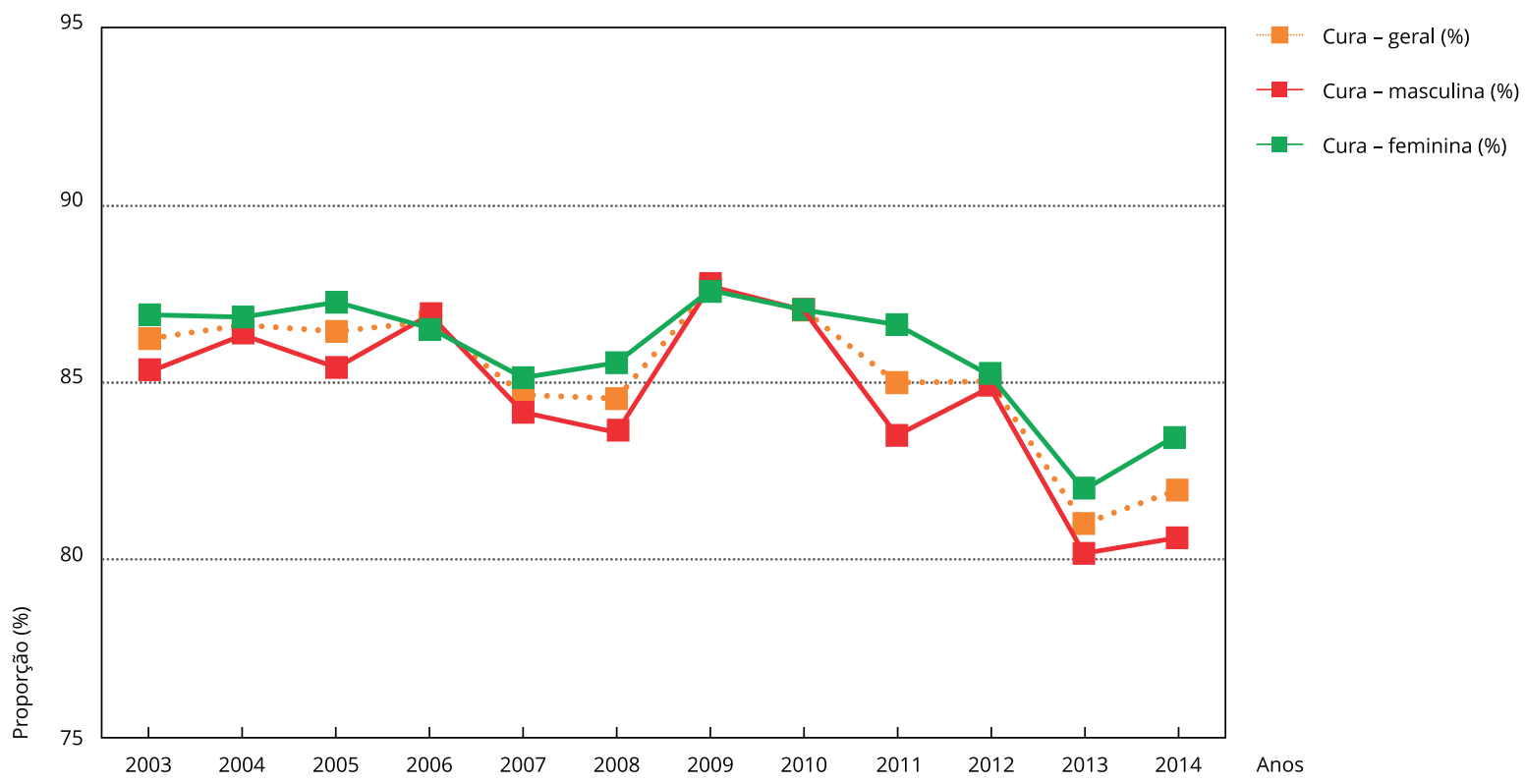

2b) Abandono

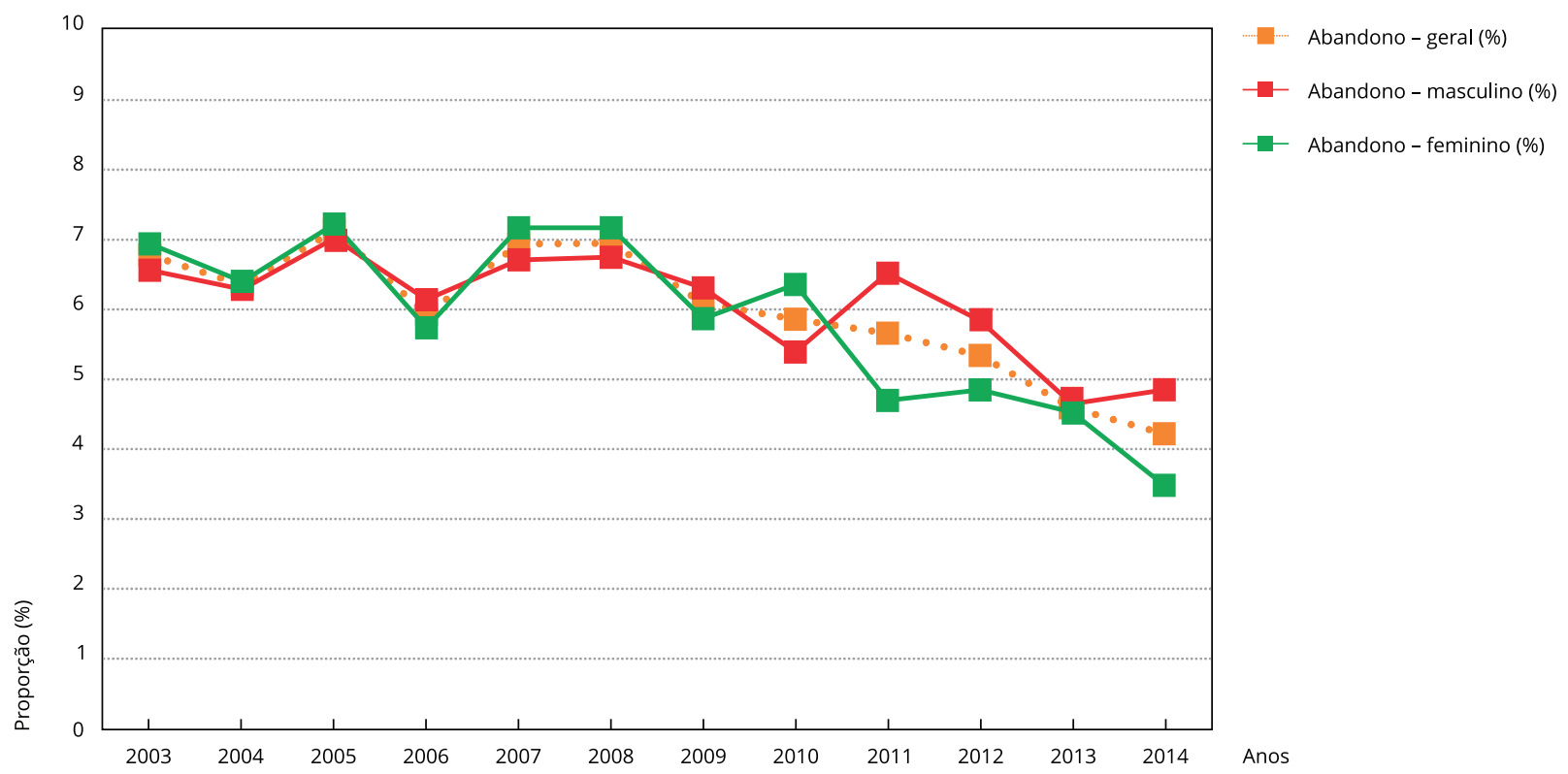


Figura 3

Proporção de casos de recidivas de hanseníase por sexo. Estado da Bahia, Nordeste do Brasil, 2001-2014.

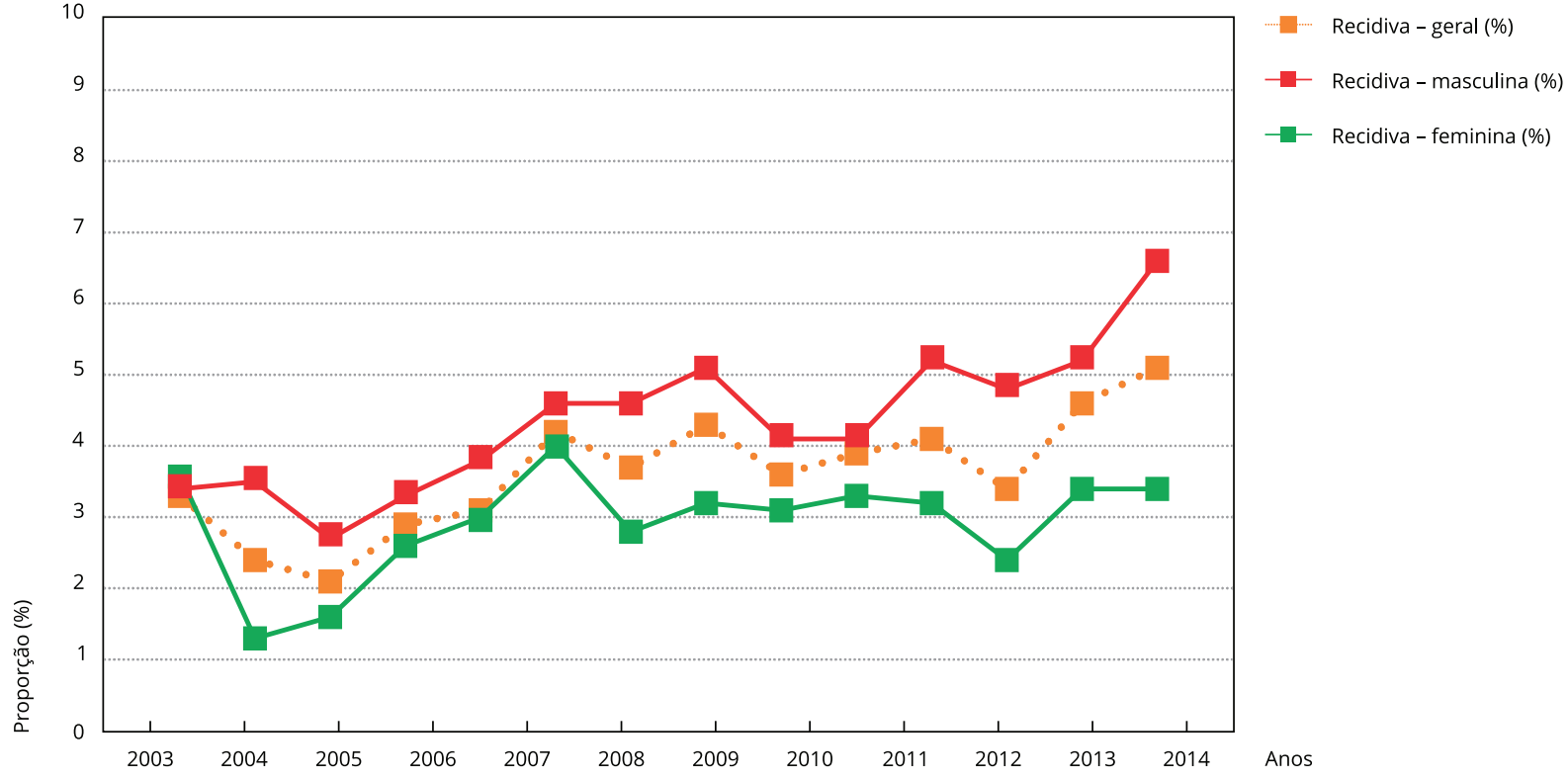

\section{Discussão}

Este estudo foi o primeiro a analisar em uma série histórica consistente de 14 anos a vulnerabilidade programática com base em indicadores operacionais da hanseníase no Estado da Bahia, incluindo a perspectiva de gênero. Os resultados indicam que é precária a atuação dos serviços de saúde no que diz respeito ao desempenho na vigilância dos contatos intradomiciliares e, quando diagnosticados casos novos, é regular a qualidade da atenção e do acompanhamento até a conclusão do tratamento.

A redução verificada em relação ao abandono é relevante, mas as entradas por recidivas superam os padrões verificados em outros estados do país, o que impõe investigação clínica e laboratorial baseando-se em fluxo estadual em unidades de referência, seguindo orientações da OMS 2 e do Ministério da Saúde 15. Essa investigação é necessária, com vistas à aplicação dos novos critérios estabelecidos para as situações que requerem retratamento, nem sempre por evento de recidiva, bem como à detecção de resistência medicamentosa ${ }^{2}$. A análise segundo gênero reforça diferenças potenciais na resolutividade dos serviços quando o caso é do sexo masculino, o que reafirma a necessidade da efetivação da política nacional de saúde do homem 16,18 .

As diretrizes para vigilância, atenção e eliminação da hanseníase que foram publicadas em fevereiro de 2016, reforçaram como ações prioritárias a detecção de novos casos, o atendimento à demanda espontânea, a busca ativa de casos novos e a vigilância de contatos. Sugeriu-se que a avaliação de contatos ocorresse anualmente, durante pelo menos cinco anos 15. Portanto, reafirma que uma abordagem única ao contato não assegura a redução da transmissão da doença. De forma adicional, as novas diretrizes ampliaram o conceito de contato para além do espaço do intradomicílio, apesar de não ter deixado de modo claro os referenciais para a operacionalização desta nova orientação. O presente trabalho não capta as novas orientações contidas nessa nova definição 15

O Estado da Bahia, mesmo tendo apresentado tendência de aumento da proporção de contatos examinados entre os registrados, mantém padrão insatisfatório de avaliação, refletindo nos baixos porcentuais de entrada de casos novos com base no exame dos contatos. Esses dados revelam possíveis 
dificuldades dos serviços de saúde para desenvolver as ações de vigilância do contato, mesmo após a ampliação da cobertura da atenção básica no estado nos últimos anos 22 . Ao longo da série histórica foram quase 50 mil contatos não avaliados, realidade semelhante a outras pesquisas que apontaram para precariedade deste indicador ${ }^{13,23}$. Um estudo realizado no Município de Cuiabá, Mato Grosso, verificou dificuldades no acesso dos contatos intradomiciliares ao exame, sendo que, entre os motivos identificados, foram verificadas: centralização da realização de exames de baciloscopia, ausência de atividades educativas e falta de sistematização no agendamento da consulta 24 . A OMS reafirmou ser um dos desafios para a eliminação da hanseníase até 2020 no nível subnacional o reconhecimento da doença, a educação e o exame qualificado de contatos 1.

Verificou-se que a proporção de cura entre os casos de hanseníase na coorte insere o Estado da Bahia no parâmetro regular, segundo diretrizes adotadas pelo Ministério da Saúde 15, revelando fragilidades no seguimento das pessoas acometidas até a conclusão do tratamento. Em 2014, a proporção de cura foi inferior à média nacional (83\%) e de cidades do Paraná e de Minas Gerais 25,26, por exemplo, apresentando tendência significativa de piora.

A política de descentralização das ações de controle da hanseníase traz para a atenção primária à saúde a responsabilidade deste processo na perspectiva da integralidade em territórios. Em 2014, a cobertura estimada da população pela estratégia de saúde da família na Bahia era de $68,9 \% 22$, no entanto, a ampliação ocorrida na série histórica em análise não impactou na melhoria deste indicador operacional de cura. Um estudo realizado para avaliar a atenção primária no tocante à hanseníase no Município de Betim, Minas Gerais, em 2015, verificou fragilidades aos atributos de acesso, orientação comunitária e orientação profissional. Constatou-se a necessidade de realizar novos trabalhos para compreender o que leva os usuários com hanseníase a não buscarem prioritariamente a rede de atenção primária 26.

O abandono do tratamento nesta série de 14 anos apresentou tendência de redução a partir de 2008 e, segundo os parâmetros do Ministério da Saúde, foi classificado no parâmetro de boa situação, com valores inferiores a $10 \% 15$. Uma das causas associadas a não adesão ao tratamento específico da hanseníase em um distrito da Índia remetia aos efeitos colaterais dos medicamentos utilizados 27 . Em revisão sistemática realizada em 2011, foram verificados poucos estudos sobre essa temática, sendo que aqueles identificados, nomearam como fatores explicativos a distância entre a casa da pessoa com hanseníase e o serviço de saúde, a duração do tratamento e a falta de um melhor acompanhamento pelos profissionais de saúde 28. Em outro estudo, sentimentos observados junto às pessoas com a doença, tais como irritação, silêncio, estigma e aversão, foram referidos como contribuintes para a baixa adesão ao tratamento em um centro de referência na Região Nordeste do Brasil 29. Entre usuários com hanseníase em tratamento irregular, verificou-se associação de baixos níveis de renda e de escolaridade, sendo a maioria considerada pobre ou indigente 30 . Em toda a série histórica, foram identificadas 2.730 mil pessoas em abandono, tornando essencial compreender os principais fatores associados.

Seria esperado que com a queda das proporções de abandono, ocorresse incremento na proporção de cura dos casos, o que não foi observado por este estudo. Por outro lado, as proporções de recidiva mantiveram-se elevadas com tendência de crescimento significativo (superior a 2,2\%, atingindo em 2014, 5\%). Esse dado traduz a proporção de recidivas entre todas as entradas no período estudado, diferenciando-se das baixas taxas de recidiva indicadas pela OMS entre os casos que receberam alta por cura em coortes com mais de 50 mil casos paucibacilares $(1,07 \%)$ e de mais de 20 mil casos multibacilares (0,77\%), após nove anos do término da polioquimioterapia 31,32 . Os casos de recidiva caracterizam o agravamento da doença baseando-se no aumento da carga bacteriana e no grau de incapacidade física, podendo indicar falhas no tratamento e a eficácia do tratamento instituído 33 . Em um estudo realizado no Estado de Mato Grosso, os fatores preditivos identificados para a recidiva foram: condições de moradia, hábitos de vida, organizações dos serviços de saúde, formas clínicas e esquemas terapêuticos 32 .

O Brasil foi o país que notificou o maior número de casos novos com entrada como recidiva no mundo 2, o que não necessariamente traduz a realidade do país e da Bahia. No entanto, tendo em vista que os critérios para o diagnóstico não são simples, é necessário implementar a vigilância de todos os casos para maior precisão, além da utilização adequada de exames complementares específicos ${ }^{34}$. $\mathrm{Na}$ Bahia, muitos desses casos são diagnosticados e tratados fora de centros de referência, e uma minoria 
realiza baciloscopia e tem a sua avaliação do comprometimento neural registrado 35 , fatos que tornam emblemática a necessidade de se discutir a qualidade deste diagnóstico, assim como o acesso a serviços especializados de maior complexidade tecnológica regionalizados, sobretudo para municípios de pequeno porte, maioria nesse estado ${ }^{36}$. Ressalta-se ainda a verificação da presença de casos com resistência medicamentosa no país, o que torna a discussão sobre recidivas ainda mais complexa 37 .

O documento relativo à estratégia global para a hanseníase no período de 2016-2020, publicado pela OMS, orienta para a priorização das ações voltadas para as mulheres e para as crianças 1 . No entanto, a análise dos indicadores da Bahia revela a necessidade também de se desenvolver estratégias específicas para a população masculina, considerando que a tendência de crescimento da proporção de contatos examinados é de menor proporção quando o caso de hanseníase de referência é homem. Por outro lado, a tendência de redução da proporção dos casos que têm alta por cura foi maior entre os homens, já no abandono, a tendência de redução que ocorre em ambos os sexos foi maior entre as mulheres. Por fim, a proporção de casos neste estudo que inicia novo tratamento por recidiva é maior entre os homens, havendo crescimento entre estes e clara redução na tendência apresentada para as mulheres. Em um contexto latino-americano de gênero, a abordagem da população masculina demanda estratégias inovadoras e contextualizadas às diferentes realidades culturais, sociais e econômicas locais 18,38 .

As desigualdades de gênero apresentam forte influência no processo saúde-doença-cuidado, sendo que os homens se tornam mais vulneráveis ao adoecimento e a formas mais graves, não apenas pela forma de viver a sua masculinidade, mas também à inadequação dos serviços de saúde na identificação e atendimento de suas necessidades específicas de saúde 38. Desde 2009, o Brasil tem buscado instituir a Política Nacional de Atenção Integral à Saúde do Homem, com o objetivo de promover ações de saúde que contribuam significativamente para a compreensão da realidade singular masculina nos seus diversos contextos socioculturais, políticos e econômicos 39. No entanto, as políticas públicas e as práticas de saúde no interior dos serviços são, muitas vezes, divergentes 40 . Além disso, mais do que a medicalização do corpo masculino, torna-se crucial a politização e sensibilização do homem, despertando a consciência das dimensões de vulnerabilidade existentes 41. Da mesma forma, torna-se estratégico reconhecer a diversidade e as especificidades da saúde do homem por parte dos gestores e profissionais de saúde, ampliando a visibilidade desta população nos serviços de saúde, incluindo necessariamente a atenção primária 42,43 . Além do investimento em pesquisas para elucidar fatores hormonais favorecerem a mulher na proteção às doenças infecciosas, o papel do homem como consumidor dos serviços de saúde, incluindo aqueles de média e alta complexidade, deve ser amplamente discutido.

O Ministério da Saúde em agosto de 2016 publicou nota técnica informativa de alerta para o exame sistemático de hanseníase na população masculina e em idosos. Esse documento foi baseado na análise de 541.090 casos novos de hanseníase notificados no período de 2001-2013, cujos resultados mostraram chances duas vezes maiores para a ocorrência de casos multibacilares nestas duas populações. Fatores biológicos, hormonais e imunossenescência também são discutidos e reforçam esse maior risco 44,45,46. A despeito da relevância, na atenção primária à saúde os avanços são ainda limitados 47 , mantendo-se como espaço muito pouco atrativo e pouco frequentado por essa população, principalmente aqueles em idade economicamente ativa 43,48. Considerando-se que as unidades de atenção básica devem ser o lócus principal para instituir medidas de vigilância, diagnóstico e tratamento da hanseníase 15 , amplifica-se a vulnerabilidade programática, contribuindo para a ampliação das incapacidades físicas e estigma nessa população.

O olhar de gênero permite enriquecer conceitualmente as perspectivas de "necessidades de saúde", bem como diferenciar as práticas profissionais em saúde de atenção integral destinadas a homens e mulheres, particulares e concretas, para alcançar maior equidade nas políticas públicas 49 . Esse aspecto deve ser considerado principalmente em populações com maior vulnerabilidade social, situação que recorre entre aqueles com doenças negligenciadas, a exemplo da hanseníase.

As limitações do presente estudo estão associadas ao fato de se utilizar base de dados secundários, muitas vezes vinculada a não completitude e a inconsistências na quantidade e qualidade da informação ao longo do tempo e entre municípios do Estado da Bahia. A despeito dos progressos obtidos ao longo do período de observação em termos de cobertura do SINAN e da melhoria na qualidade das informações, as notificações da hanseníase podem não ter sido devidamente informadas em termos de 
precisão. Por exemplo, não foi possível avaliar a proporção de casos novos com a avaliação de incapacidade física pelos baixos porcentuais de registro no momento da alta. A interpretação dos dados de tendência e as diferenças verificadas por gênero devem considerar essas circunstâncias. No entanto, o fato de ser uma série histórica de 14 anos de um estado como a Bahia, com poucas evidências disponibilizadas, justifica a sua plena utilização.

\section{Conclusão}

O presente estudo aponta para fragilidades dos serviços de saúde no Estado da Bahia em desenvolver ações de controle, sobretudo aquelas vinculadas à vigilância dos contatos intradomiciliares, diagnóstico e tratamentos oportunos, além de seguimento das pessoas acometidas pela hanseníase. Reconheceu-se a ampliação da dimensão de vulnerabilidade programática quando se considera a população masculina, em especial para os aspectos relativos à cura e à recidiva.

Novas tecnologias devem ser instituídas para o controle das doenças negligenciadas, a exemplo de novos fármacos, vacinas e meios diagnósticos 19 . No entanto, a ampliação do acesso, a melhoria da qualidade das ações de controle e o planejamento diferenciado de estratégias com foco nos homens devem ser instituídos. Da mesma forma, é necessário rever a qualidade da vigilância dos eventos de recidiva, a fim de verificar se de fato correspondem à falência terapêutica.

As reflexões sobre diferentes dimensões de vulnerabilidades, incluindo as questões de gênero, devem ser consideradas no planejamento e desenvolvimento de ações de controle, principalmente por ser um evento negligenciado. Outros estudos são necessários para avaliar possíveis barreiras, qualidade das ações desenvolvidas e acesso às ações, principalmente nas periferias das grandes cidades e nos municípios de pequeno porte, neste último caso, tanto em regiões urbanas quanto rurais.

\section{Colaboradores}

E. A. Souza e A. N. Ramos Jr. contribuíram na concepção do projeto, análise e interpretação dos dados, redação do artigo e revisão crítica relevante do conteúdo intelectual, e aprovação final da versão a ser publicada. R. N. Boigny, A. F. Ferreira, C. H. Alencar e M. L. W. Oliveira colaboraram na análise e interpretação dos dados, redação do artigo e revisão crítica relevante do conteúdo intelectual, e aprovação final da versão a ser publicada.

\section{Agradecimentos}

Suporte financeiro do Conselho Nacional de Desenvolvimento Científico e Tecnológico (CNPq), processo número 404505/2012-0, Edital MCTI/ CNPq/MS-SCTIE-Decit no 40/2012 - Pesquisa em Doenças Negligenciadas. E. A. Souza foi bolsista de doutorado da Coordenação de Aperfeiçoamento de Pessoal de Nível Superior (Capes). 


\section{Referências}

1. World Health Organization. Global leprosy strategy 2016-2020: accelerating towards a leprosy-free world. http://apps.searo.who. int/PDS_DOCS/B5233.pdf (acessado em 07/ Out/2016)

2. World Health Organization. Global leprosy update, 2015: time for action, accountability and inclusion. Wkly Epidemiol Rec 2016; 91:405-20.

3. Alencar CHM. Padrões epidemiológicos da hanseníase em área de alto risco de transmissão nos estados do Maranhão, Pará, Tocantins e Piauí: 2001-2009 [Tese de Doutorado]. Fortaleza: Departamento de Saúde Comunitária, Universidade Federal do Ceará; 2011.

4. Barbosa JC, Ramos Jr. AN, Alencar OM, Pinto MSP, Castro CGJ. Leprosy after release from treatment in the Brazilian Unified Health System: aspects for access in the Northeast region. Cad Saúde Colet (Rio J.) 2014; 22:351-8.

5. Noriega LF, Chiacchio ND, Noriega AF, Pereira GA, Vieira ML. Leprosy: ancient disease remains a public health problem nowadays. An Bras Dermatol 2016; 91:547-8.

6. Hofstraat K, van Brakel WH. Social stigma towards neglected tropical diseases: a systematic review. Int Health 2016; 8:53-70.

7. Freitas LR, Duarte EC, Garcia LP. Trends of main indicators of leprosy in Brazilian municipalities with high risk of leprosy transmission, 2001-2012. BMC Infect Dis 2016; 16:472.

8. Monteiro LD, Martins-Melo FR, Brito AL, Alencar CH, Heukelbach J. Physical disabilities at diagnosis of leprosy in a hyperendemic area of Brazil: trends and associated factors. Lepr Rev 2015; 86:240-50.

9. Martins PV, Iriart JAB. Itinerários terapêuticos de pacientes com diagnóstico de hanseníase em Salvador, Bahia. Physis (Rio J.) 2014; 24:273-89.

10. Pinto Neto JM, Villa TCS, Oliveira M, Barbeira C. O controle dos comunicantes de hanseníase no Brasil: uma revisão de literatura. Hansen Int 2000; 25:163-76.

11. Smith W, Aerts A. Role of contact tracing and prevention strategies in the interruption of leprosy transmission. Lepr Rev 2014; 85:2-17.

12. Andrade AR, Grossi MA, Buhrer-Sekula S, Antunes CM. Seroprevalence of ML Flow test in leprosy contacts from State of Minas Gerais, Brazil. Rev Soc Bras Med Trop 2008; 41:56-9.

13. Lobato DC, Neves DCO, Xavier MB. Avaliação das ações da vigilância de contatos domiciliares de pacientes com hanseníase no Município de Igarapé-Açu, Estado do Pará, Brasil. Revista Pan-Amazônica de Saúde 2016; 7:45-53.

14. Ministério da Saúde. Nota informativa conjunta - alerta para o exame sistemático de hanseníase na população masculina e em idosos. http://portalsaude.saude.gov.br/images/ pdf/2016/setembro/06/Nota-InformativaConjunta-n---01--SAS-e-SVS--para-publica---o.pdf. (acessado em 09/Nov/2016).
15. Departamento de Vigilância das Doenças Transmissíveis, Secretaria de Vigilância em Saúde, Ministério da Saúde. Diretrizes para vigilância, atenção e eliminação da hanseníase como problema de saúde pública. Manual técnico-operacional. Brasília: Ministério da Saúde; 2016.

16. Ayres JRCM, França Júnior I, Calazans GJ, Saletti Filho HC. O conceito de vulnerabilidade e as práticas de saúde: novas perspectivas e desafios. In: Czeresnia D, Freitas CM, organizadores. Promoção da saúde: conceitos, reflexões, tendências. Rio de Janeiro: Editora Fiocruz; 2003. p. 117-40.

17. Mendes EV. As redes de atenção à saúde. Brasília: Organização Pan-Americana da Saúde; 2011.

18. Pinheiro RS, Viacava F, Travassos C, Brito AS. Gênero, morbidade, acesso e utilização de serviços de saúde no Brasil. Ciênc Saúde Coletiva 2002; 7:687-707.

19. Instituto de Pesquisa Econômica Aplicada. Atlas do desenvolvimento humano no Brasil 2013. http://atlasbrasil.org.br/2016/perfil/ bahia2013 (acessado em 01/Set/2016).

20. Andrade Schramm JM, Campos MR, Emmerick ICM, Pereira Mendes LV, Mota JC, Silva Junior SHA. Spatial analysis of neglected diseases in Brazil, 2007 to 2009. Tempus (Brasília) 2016; 10:119-42.

21. Kim HJ, Fay MP, Feuer EJ, Midthune DN. Permutation tests for joinpoint regression with applications to cancer rates. Stat Med 2000; 19:335-51.

22. Ministério da Saúde. Histórico de cobertura da saúde da família. http://dab.saude.gov. br/portaldab/historico_cobertura_sf.php (acessado em 02/Set/2016).

23. Dessunti EM, Soubhia Z, Alves E, Aranda CM, Barro MPAA. Hanseníase: o controle dos contatos no Município de Londrina - PR em um período de dez anos. Rev Bras Enferm 2008; 6:689-93.

24. Oliveira SP. Acessibilidade ao exame de contato de hanseníase na Estratégia de Saúde da Família em Cuiabá, Mato Grosso - Brasil [Dissertação de Mestrado]. Salvador: Instituto de Saúde Coletiva, Universidade Federal da Bahia; 2013.

25. Oliveira KS, Souza J, Campos RB, Zilly A, Silva-Sobrinho RA. Evaluation of leprosy epidemiological and operational indicators in priority municipalities in the state of Paraná, 2001 to 2010. Epidemiol Serv Saúde 2015; 24:507-16.

26. Vieira NF. Avaliação da atenção primária à saúde nas ações de controle da hanseníase no Município de Betim, Minas Gerais [Dissertação de Mestrado]. Belo Horizonte: Escola de Enfermagem, Universidade Federal de Minas Gerais; 2015. 
27. Kumar A, Girdhar A, Chakma JK, Girdhar BK WHO multidrug therapy for leprosy: epidemiology of default in treatment in Agra district, Uttar Pradesh, India. Biomed Res Int 2015; 2015:705804.

28. Heukelbach J, André Chichava O, Oliveira AR, Häfner K, Walther F, Alencar CH, et al. Interruption and defaulting of multidrug therapy against leprosy: population-based study in Brazil's Savannah Region. PLoS Negl Trop Dis 2011; 5:e1031.

29. Silva CA, Albuquerque VL, Antunes MF. Leprosy as a neglected disease and its stigma in the Northeast of Brazil. Indian J Lepr 2014; 86:539.

30. Lopes VAS, Rangel EM. Hanseníase e vulnerabilidade social: uma análise do perfil socioeconômico de usuários em tratamento irregular. Saúde Debate 2014; 38:817-29.

31. Risk of relapse in leprosy. The Leprosy Unit, WHO. Indian J Lepr 1995; 67:13-26.

32. Ferreira SMB, Ignotti E, Gamba MA. Fatores associados à recidiva em hanseníase em Mato Grosso. Rev Saúde Pública 2011; 45:756-64.

33. Ferreira SMB, Ignotti E, Gamba MA. Clinical and laboratory characteristics in the retreatment of leprosy relapse. Rev Bras Epidemiol 2012; 15:573-81.

34. Kaimal S, Thappa DM. Relapse in leprosy. Indian J Dermatol Venereol Leprol 2009; 75:126.

35. Silva FL. Recidiva da hanseníase no Estado da Bahia [Dissertação de Mestrado]. Salvador: Instituto de Saúde Coletiva, Universidade Federal da Bahia; 2015.

36. Alencar CH, Ramos Jr. AN, Sena Neto SA, Murto C, Alencar MJ, Barbosa JC, et al. Diagnóstico da hanseníase fora do município de residência: uma abordagem espacial, 2001 a 2009. Cad Saúde Pública 2012; 28:1685-98.

37. Silva Rocha A, Cunha M, Diniz LM, Salgado C, Aires MA, Nery JA, et al. Drug and multidrug resistance among Mycobacterium leprae isolates from Brazilian relapsed leprosy patients. J Clin Microbiol 2012;50:1912-7.

38. Figueiredo W. Assistência à saúde dos homens: um desafio para os serviços de atenção primária. Ciênc Saúde Coletiva 2005; 10:105-9.

39. Departamento de Ações Programáticas Estratégicas, Secretaria de Atenção à Saúde, Ministério da Saúde. Política Nacional de Atenção Integral à Saúde do Homem (princípios e diretrizes). http://portalsaude.saude.gov.br/ images/pdf/2014/maio/21/CNSH-DOCPNAISH---Principios-e-Diretrizes.pdf (acessado em 01/Set/2016)
40. Schraiber LB. Necessidades de saúde, políticas públicas e gênero: a perspectiva das práticas profissionais. Ciênc Saúde Coletiva 2013; 17:2635-44.

41. Carrara S, Russo JA, Faro L. A política de atenção à saúde do homem no Brasil: os paradoxos da medicalização do corpo masculino. Physis (Rio J.) 2009; 19:659-78.

42. Gomes R, Moreira MCN, Nascimento EF, Rebello LEFS, Couto MT, Schraiber LB. Os homens não vêm! Ausência e/ou invisibilidade masculina na atenção primária. Ciênc Saúde Coletiva 2011; 16:983-992.

43. Alves RF, Silva RP, Ernesto MV, Lima AGB, Souza FM. Gênero e saúde: o cuidar do homem em debate. Psicol Teor Prat 2011; 13:152-66.

44. Nobre ML. Estratégias para bloquear a transmissão da hanseníase em município hiperendêmico - Mossor, RN [Tese de Doutorado]. Rio de Janeiro: Instituto Oswaldo Cruz, Fundação Oswaldo Cruz; 2016.

45. Morales-Montor J, Chavarria A, De León MA, Del Castillo LI, Escobedo EG, Sánchez EN, et al. Host gender in parasitic infections of mammals: an evaluation of the female host supremacy paradigm. J Parasitol 2004; 90:531-46.

46. Youm YH, Kanneganti TD, Vandanmagsar B, Zhu X, Ravussin A, Adijiang A, et al. The Nlrp3 inflammasome promotes age-related thymic demise and immunosenescence. Cell Rep 2012; 1:56-68.

47. Oliveira MM, Daher DV, da Silva JLL, de Araújo Andrade SSC. A saúde do homem em questão: busca por atendimento na atenção básica de saúde. Ciênc Saúde Coletiva 2015; 20:273-8.

48. Moreira MCN, Gomes R, Ribeiro CR. E agora o homem vem! Estratégias de atenção à saúde dos homens. Cad Saúde Pública 2016; 32:e00060015.

49. Knauth DR, Couto MT, Figueiredo WS. A visão dos profissionais sobre a presença e as demandas dos homens nos serviços de saúde: perspectivas para a análise da implantação da Política Nacional de Atenção Integral à Saúde do Homem. Ciênc Saúde Coletiva 2012; 17:2617-26. 


\section{Abstract}

The aim of this study was to analyze operational indicators and time trends in leprosy control from a gender perspective in Bahia State, Brazil, from 2001 to 2014. This was a time series study based on epidemiological data on leprosy from the Brazilian National System of Diseases of Notification, using joinpoint Poisson and polynomial regression. Of the 40,054 new cases of leprosy, 47.1\% of the recorded contacts were not examined, with a significant upward trend, especially in women (average annual percentage change $-A A P C=$ 5.6; 95\%CI: 3.5; 7.7) when compared to men $(A A P C=3.0 ; 95 \% C I: 0.5 ; 5.6)$. The proportion of cure in the 2003-2014 cohort was 85\%, with a downward trend, especially in men $(A A P C=-0.5$; 95\%CI: - 0.9; 0.0), compared to women (AAPC = -0.4; 95\%CI: -0.7; -0.1). Treatment dropout rate was $5.5 \%$, with a more significant downward trend in women $(A A P C=-4.9 ; 95 \% C I:-8.7 ;-1.1)$ than in men (AAPC $=-2.7 ; 95 \% \mathrm{CI}:-4.4 ;-1.0)$. Relapse was recorded in $3.8 \%$ of all the entries during the same period; women showed a significant downward trend $(A A P C=-2.2 ; 95 \% C I:-3.3 ;-1.0)$ and men a significant upward trend $(A A P C=4.9$; 95\%CI: 2.9; 6.8). Polynomial regression analysis was consistent with joinpoint regression. Leprosy in Bahia State shows operational indicators with significant magnitude and time trends, especially in the male population. Health services' insufficient performance in conducting contact surveillance and longitudinal care reveal various dimensions of vulnerability.

Leprosy; Time Series Studies; Gender and Health

\section{Resumen}

El objetivo de este estudio fue analizar indicadores operacionales de control de la lepra y su tendencia temporal, desde la perspectiva de género, en el estado de Bahía, Brasil, de 2001 a 2014. Se trata de un estudio de serie temporal, basado en datos epidemiológicos de la lepra en el Sistema Nacional de Enfermedades de Notificación Obligatoria, con análisis de regresión de Poisson por joinpoints $y$ de regresión polinomial. De los 40.054 casos nuevos de hanseniasis analizados, un 47,1\% de los contactos registrados no fueron examinados, pero con una tendencia significativa de aumento, de forma más acentuada entre las mujeres $(A A P C=$ 5,6; IC95\%: 3,5; 7,7), en comparación a los hombres (AAPC = 3,0; IC95\%: 0,5; 5,6). La proporción de cura en la cohorte 2003-2014 fue de un 85\%, con una tendencia de reducción más acentua$d a$ en los hombres (AAPC $=-0,5$; IC95\%: $-0,9$; $0,0)$, comparados con las mujeres $(A A P C=-0,4$; IC95\%: -0,7;-0,1). La proporción de casos en abandono de tratamiento durante el período fue de un 5,5\%, con tendencia de reducción más significativa entre mujeres $(A A P C=-4,9$; IC95\%: - 8,7; - 1,1) que en hombres (AAPC $=-2,7$; IC95\%: -4,4;-1,0). La recidiva se verificó en un 3,8\% de todas las entradas del periodo; las mujeres presentaron una tendencia de reducción significativa ( $A A P C=-2,2$; IC95\%: -3,3; - 1,0) y los hombres, de crecimiento significativo $(A A P C=$ 4,9; IC95\%: 2,9; 6, 8). El análisis por regresión polinomial fue consistente con el análisis de joinpoints. La hanseniasis en el estado de Bahía presenta indicadores operacionales con magnitud $y$ tendencia temporal significativas, en especial entre la población masculina. El desempeño insuficiente de los servicios de salud al realizar la vigilancia de contactos y la longitudinalidad del cuidado revelan diferentes dimensiones de vulnerabilidad.

Lepra; Estudios de Series Temporales; Género y Salud
Recebido em 16/Nov/2016

Versão final reapresentada em 21/Mar/2017 Aprovado em 25/Abr/2017 